\title{
Rensselaer Component of the Terascale Simulation Tools and Technologies
}

\author{
Agreement No. DE-FC02-01ER25460 Final Report: \\ Institutional Faculty PI's: Mark S. Shephard and Joseph E. Flaherty \\ Associated Faculty: Keneth E. Jansen \\ Research Associate: Andy Bauer \\ Affiliated Post Doc: Luzhong Yin, Suleyman Kocak, S. Lankalapalli, Yunhua Luo \\ Research Engineers: Dinesh Godavarty, Christophe Dupre \\ Affiliated Students: Eunyoung Seol, X. Luo, Mohan Nuggehally, Jie Wan
}

\section{Introduction}

The Terascale Simulation Tools and Technologies (TSTT) SciDAC center focused on the development and application on SciDAC applications of advanced technologies to support unstructured grid simulations. As part of the TSTT team the RPI group focused on developing automated adaptive mesh control tools and working with SciDAC accelerator and fusion applications on the use of these technologies to execute their simulations. The remainder of this report provides a brief summary of the efforts carried out by the RPI team to support SciDAC applications (Section 2) and to develop the TSTT technologies needed for those automated adaptive simulations (Section 3). More complete information on the technical developments can be found in the cited references and previous progress reports.

\section{Applications Developments:}

\subsection{Accelerator Modeling with SLAC National Accelerator Laboratory}

In order to perform the analyses needed in the design of next generation accelerators, researchers at the SLAC National Accelerator Laboratory have been developing higher order parallel finite element methods. High-order methods are well known to provide higher rates of convergence, which can provide SciDAC applications with an effective means to address critical applications with dramatically reduced levels of computational effort. Two areas where the RPI TSTT team bas contributed to SLAC efforts are the development of parallel adaptive mesh control loops and tool to produce curved finite element mesh entities as needed to properly apply high order methods on domains defined as CAD models.

The combination of high required accuracy and geometric complexity of full accelerator cavity assemblies requires the execution of automated mesh adaptation that operates in parallel. The RPI TSTT team developed parallel adaptive tools to integrate with the parallel version of OMEGA3P. The key tools/developments included a parallel mesh database [25-27,29], parallel dynamic load balancing [29], and parallelization of mesh adaptation procedures $[1,24]$, solution transfer functions and error estimation modules. These procedures effectively supported simulations providing accurate solution results $[11,15,18]$. 
A complexity that arises when applying higher order finite elements to complex 3-D domains is the need to have curved finite elements. The common approach to the construction of such meshes is to take advantage of available mesh generators that perform straight-sided mesh generation and then curve the mesh edges and faces adjacent to curved domain boundaries to conform to the boundaries. However, the resulting meshes often contain invalid elements because curving the mesh entities to conform to boundaries can lead to negative Jacobian determinants in the closures of elements. To address this problem, the RPI TSTT team has been developing mesh curving techniques that to be able to produce and control curved meshes [19,20,37]

\subsection{Fusion Modeling with PPPL}

The RPI TSTT team has been working with the Steve Jardin's group at PPPL to create accurate MHD simulation technologies. Initial efforts looked into extending adaptive discontinuous Galerkin formulations to solve MHD problems [14]. As the PPPL group moved more heavily to the use of high order finite element methods we investigated the potential advantages of stabilized high-order finite element formulations [9]. As the PPPL group began to develop their new high-order code M3D-C1, the RPI TSTT became directly involved with developing the structures needed to support parallel mesh adaptation and to construct the 2-D parallel adaptive mesh control tools needed $[2,12]$.

\section{TSTT Technology Development}

\subsection{Mesh Interface}

The TSTT team has defined a mesh interface to support unstructured meshes based on entities and their adjacencies [5]. The RPI team was involved in its design and implementation $[8,22]$. The RPI implementation of the TSTT mesh interface builds on its research and development of flexible mesh databases that can store the desired mesh adjacencies [26]. The RPI implementation was fully implemented to support parallel distributed meshes [25,27,29,33].

\subsection{Geometry Interface}

The geometric domain is a central part of the high level definition of a simulation problem. In today's environments the key sources of these domain definitions are CAD models, meshes (simulation meshes and STL files) and image data. To effectively support general simulation capabilities, including automated adaptive analysis and evolving geometry problems, it is necessary that a high level understanding of the domain definition be used. The concept of geometric model topological entities and their adjacencies provide a natural abstraction for supporting these needs. A set of functions have been defined and implemented to support the interactions of the simulation process with general non-manifold geometric domains [3,4,31].

\subsection{Mesh Adaptation Service}


Adaptive methods are central to ensuring the reliability of the simulations used for SciDAC applications. An area of emphasis of the RPI TSTT team has been the development a service to that can be directly integrated with SciDAC analysis procedures to provide adaptive simulations that can greatly increase the reliability of the results obtained. The service that has been developed to support generalized mesh adaptation by controlling the mesh size through the domain of interest [16]. To ensure the ability to deal with general curved geometries that can come from CAD systems, the procedures build on a generalized interaction with the geometric model and ensure the mesh can properly represent the domain of interest $[17,19,20,30]$. The mesh adaptation service, which fully operates in parallel $[1,29,32]$, has been used to develop adaptive simulations for accelerator and fusion simulations as discussed in Section 2. It has also been uesd to support several other applications including ones with evolving geometries [35,36], and ones requiring anisotropic mesh adaptation $[6,21,28]$. Some of methods and ideas used in the development of the mesh adaptation service are being used to support adaptive multiscale simulations $[7,34]$.

\section{Publications:}

[1] Alauzet, F., Li, X., Seol, E.,S. and Shephard, M.S., Parallel Anisotropic 3D Mesh Adaptation by Mesh Modification, Engineering with Computers, 21(3):247-258, 2006.

[2] A.C. Bauer, M.S. Shephard, S.C. Jardin, "Development of an Adaptive Loop for Extended MHD Simulations", Proc. 7th US Nat. Congress on Comp. Mech., p. 1153, 2006.

[3] Beall, M.W., Walsh, J. and Shephard, M.S, "A comparison of techniques for geometry access related to mesh generation", Engineering with Computers, 20(3):210-221, 2004.

[4] Beall, M.W., Walsh, J. and Shephard, M.S., "Accessing CAD geometry for mesh generation", 12th International Meshing Roundtable, Sandia National Laboratories, SAND-2003-3030P, pp. 33-42, 2003.

[5] Beall, M.W. and Shephard, M.S., "A general topology-based mesh data structure", Int. J. Numer. Meth. Engng., 40(9):1573-1596, 1997.

[6] N. Chevaugeon, P. Hu, X. Li, J. Xin, D. Cler, J.E. Flaherty and M.S. Shephard, "Discontinuous Galerkin methods applied to shock and blast problems", Journal of Scientific Computing, 22:227-243, 2005.

[7] Datta, D., Picu, R.C. and Shephard, M.S., "Composite Grid Atomistic Continuum Method: An adaptive approach to bridge continuum with atomistic analysis", Journal of Multiscale Computational Engineering, 2(3):401-420, 2004.

[8] L. Diachin, A. Bauer, B. Fix, J. Kraftcheck, K. Jansen, X. Luo, M. Miller, C. Ollivier-Gooch, M. S. Shephard, T. Tautges and H. Trease, "Interoperable mesh and geometry tools for advanced petascale simulations", Journal of Physics: Conference Series, 78-012015,6 pages, 2007.

[9] Evans, J.A., Jansen, K.E., Shephard, M.S. and Bauer, A.C., “A multiscale stabilization of the streamfunction form of the steady state Navier-Stokes equations", 
Proc. SciDAC 2006, DOE, Journal of Physics: Conference Series, 46, pp. 463-467, 2006.

[10]Flaherty, J.E., Krivodonova, L., Remacle, J.-F. and Shephard, M.S., "Aspects of discontinuous Galerkin methods for hyperbolic conservation laws" Finite Elements in Analysis and Design, 38:889-908, 2002.

[11] Ge, L., Lee, L, Zenghai, L., Ng, C., Ko, K., Luo, Y and Shephard, M.S., “Adaptive Mesh Refinement for High Accuracy Wall Loss Determination in Accelerating Cavity Design", IEEE Conf. on Electromagnetic Field Computations, June, 2004.

[12] S. C. Jardin, N. Ferraro, J. Breslau, A. Bauer, M.S. Shephard, Two-fluid ExtendedMHD Calculations of Toroidal Equilibrium and Collisionless Reconnection in Magnetized Plasmas, European Physical Society meeting on Plasma Physics, July, 26, 2007.

[13]Ives, L., Bui, T., Vogler, W., Bauer, A., Shephard, M., and Beall, M., "Operation and performance of a 3D finite element charged particle code with adaptive meshing Source", 5Th IEEE Int. Vacuum Electronics Conf., IVEC 2004, pp. 316-317, 2004.

[14] S. Lankalapalli, J.E. Flaherty, M.S. Shephard, H. Strauss and S.C. Jardin, "An Adaptive Finite Element Method for Magnetohydrodynamics", Journal of Computational Physics, 225(1):363-381, 2007

[15] L.-Q. Lee, V. Akcelik, S. Shen, L. Ge, E. Prudencio, G. Schussman, R. Uplenchwar, C. Ng, K. Ko, X-J. Luo and M.S. Shephard, "Enabling technologies for petascale electromagnetic accelerator simulation", Journal of Physics: Conference Series, 78012040, 5 pages, 2007.

[16]Li, X., Shephard, M.S. and Beall, M.W., “3-D Anisotropic Mesh Adaptation by Mesh Modifications", Comp. Meth. Appl. Mech.Engng., 194(48-49):4915-4950, 2005.

[17]Li, X., Shephard, M.S. and Beall, M.W., "Accounting for curved domains in mesh adaptation", International Journal for Numerical Methods in Engineering, 58:246276, 2003.

[18]Luo, Y., Malik, I., Li, Z., Shephard, M.S. and Ko, K., “Adaptive mesh refinement in accelerator modeling with OMEGA3P", Proc. 7th US Nat. Congress on Comp. Mech., ISBN: 0-9743254-0-6, pp. 50, 2003.

[19]Luo, X.-J., Shephard, M.S., O’Bara, R.M., Nastasia, R. and Beall, M.W., “Automatic p-version mesh generation for curved domains", Engineering with Computers, 20(3):273-285, 2004.

[20]Luo, X., Shephard, M.S., Remacle, J.-F., O’Bara, R.M., Beall, M.W., Szabó, B.A. and Actis, R., "p-Version Mesh Generation Issues", 11th International Meshing Roundtable, pp. 343-354, 2002.

[21] J. Mueller, O. Sahni, K. E. Jansen, M. S. Shephard and C. A. Taylor, "A tool for the efficient FE-simulation of cardio-vascular flow", Proc. 2nd NAFEMS CFD Seminar: Simulation of Complex Flows (CFD), 2005.

[22] Ollivier-Gooch, C., Chand, K., Dahlgren, T., Diachin, L., Fix, B., Kraftcheck, J., Li, X., Seol. E.S., Shephard, M.S., Tautges, T. and Trease, H., “The TSTT Mesh Interface", 44th AIAA Aerospace Sciences Meeting and Exhibit, AIAA 2006-529, Reno Nevada, Jan. 2006. 
[23] Remacle, J.-F., Li, X., Shephard, M.S. and Flaherty, J.E., “Anisotropic Adaptive Simulation of Transient Flows using Discontinuous Galerkin Methods",

International Journal for Numerical Methods in Engineering, 62:899-923, 2005.

[24] Remacle, J.-F., Li, X, Chevaugeon, N. and Shephard, M.S., "Transient mesh adaptation using conforming and non-conforming mesh modifications", 11th International Meshing Roundtable, pp. 261-272, 2002.

[25] Remacle, J.-F., Klass, O., Flaherty, J.E. and Shephard, M.S., "A parallel algorithm oriented mesh database", Engineering with Computers, 18(3):274-284, 2002.

[26] Remacle, J.-F. and Shephard, M.S., "An algorithm oriented mesh database", International Journal for Numerical Methods in Engineering, 58:349-374, 2003.

[27] Remacle, J.-F., Shephard, M.S., Flaherty, J.E. and Klaas, O., "Parallel Algorithm oriented mesh database", Proc. 10th International Meshing Roundtable, Report No. SAND 2001-2976C, pp. 341-349, 2001.

[28] Sahni, O. Mueller, J., Jansen, K.E., Shephard, M.S. and Taylor, C.A., "Efficient Anisotropic Adaptive Discretization of Cardiovascular System", Comp. Meth. Appl. Mech. Engng., 195(41-43):5634-5655, 2006

[29] Seol, E.S. and Shephard, M.S., "Efficient distributed mesh data structure for parallel automated adaptive analysis", Engineering with Computers, 22(3-4):197-213, 2006.

[30] Shephard, M.S., Flaherty, J.E., Jansen, K.E., Li, X., Luo, X.-J., Chevaugeon, N., Remacle, J.-F., Beall, M.W. and O'Bara, R.M., "Adaptive mesh generation for curved domains", J.for Applied Numerical Mathematics, 53(2-3)251-271, 2005.

[31] Shephard, M.S., Beall, M.W., O’Bara, R.M. and Webster, B.E., “Toward simulationbased design", Finite Elements in Analysis and Design, 40:1575-1598, 2004.

[32] M.S. Shephard, K.E. Jansen, O. Sahni and L.A. Diachin, "Parallel Adaptive Simulations on Unstructured Meshes", Journal of Physics: Conference Series, 78012053, 10 pages, 2007.

[33] M. S. Shephard and E. S. Seol, "Flexible Distributed Mesh Data Structure for Parallel Adaptive Analysis", Advanced Computational Infrastructures for Parallel and Distributed Adaptive Applications, Wiley, to appear.

[34] M. S. Shephard, E. S. Seol and B. FrantzDale, "Toward a Multi-Model Hierarchy to Support Multiscale Simulations", Handbook of Dynamic System Modeling, P.A. Fishwick, Editor, Chapman \& Hall, Boca Raton, pp. 12.1-12.18, 2007.

[35] Wan, J., Kocak, S. and Shephard, M.S., “Automated adaptive 3-D forming simulation process", Engineering with Computers, 21(1):47-75, 2005.

[36] Wan, J., Kocak, S. and Shephard, M.S., "Automated adaptive forming simulations", 12th International Meshing Roundtable, Sandia National Laboratories, SAND-20033030P, pp. 323-334, 2003.

[37] Yin, L., Shephard, M.S., Luo, X., "Identifying and Meshing Thin Sections of 3-d Curved Domains", Proc. 14th International Meshing Roundtable, Springer, pp. 33$54,2005$. 